\title{
Nuclear Neuroimaging in Social Anxiety Disorder: A Review
}

\author{
Alexander G. Doruyter ${ }^{1}$, Patrick Dupont ${ }^{2}$, Dan J. Stein ${ }^{3}$, Christine Lochner ${ }^{4}$, and James M. Warwick ${ }^{1}$ \\ ${ }^{I}$ Division of Nuclear Medicine, Stellenbosch University, Cape Town, South Africa; ${ }^{2}$ Laboratory of Cognitive Neurology, \\ KU Leuven, Leuven, Belgium; ${ }^{3}$ MRC Unit on Risk and Resilience in Mental Disorders, University of Cape Town, Cape Town, \\ South Africa; and ${ }^{4}$ MRC Unit on Risk and Resilience in Mental Disorders, Stellenbosch University, Cape Town, South Africa
}

\begin{abstract}
In psychiatric research, nuclear imaging complements MRI. A recent neuroimaging review of social anxiety disorder focused predominantly on MRI, omitting the contribution of nuclear imaging methods. Nuclear imaging investigations of neural activity are sparse but have generally yielded results consistent with studies performed using MRI. Evidence for disturbances in neurotransmitter systems in social anxiety disorder is limited but suggestive of both serotonergic and dopaminergic dysfunction. Research focusing on additional molecular targets using existing and novel tracers, combined with recent technologic innovations and trends in collaborative methodology, may shape future nuclear imaging endeavors in this field.
\end{abstract}

Key Words: social anxiety disorder; positron emission tomography; single photon emission computed tomography; functional neuroimaging; molecular imaging

J Nucl Med 2018; 59:1794-1800

DOI: 10.2967/jnumed.118.212795

\section{$\mathbf{S}$} anxiety in social or performance situations and a marked fear of negative evaluation and embarrassment, leading to significant distress and functional impairment (1). Epidemiologic data demonstrate that the condition is common (2) and is frequently associated with severe functional impairment and morbidity (3). There is evidence that both genetic and environmental factors play a role in the pathogenesis of SAD (4), but knowledge of the neurobiologic processes involved in the disorder and how therapies exert their effects is still far from complete.

Neuroimaging modalities represent valuable tools to investigate brain function in psychiatric disorders, including SAD. Several excellent reviews and metaanalyses of functional neuroimaging have consistently highlighted hyperactivity in limbic and paralimbic structures (5-7) and have led to a proposed network model of SAD (7). Of these reviews, only the earlier two included nuclear neuroimaging studies, whereas the latest focused exclusively on MRI-based work. Several additional nuclear imaging experiments have been conducted since the publication of these reviews. The aim of this paper is to summarize the contribution of nuclear

Received Apr. 11, 2018; revision accepted Oct. 3, 2018.

For correspondence or reprints contact: Alexander Doruyter, Division of Nuclear Medicine, Tygerberg Hospital, Francie Van Zijl Dr., Tygerberg, 7505, Western Cape, South Africa.

E-mail: doruyter@sun.ac.za

Published online Oct. 5, 2018.

COPYRIGHT (C) 2018 by the Society of Nuclear Medicine and Molecular Imaging. neuroimaging to the SAD literature, complementing recent work that has focused on MRI-based experiments.

\section{MATERIALS AND METHODS}

Relevant literature was identified using the search terms ("social phobia" OR "social anxiety") AND ("SPECT" OR "SPET" OR "single photon" OR "positron" OR "PET") in PubMed (https://www.ncbi. nlm.nih.gov/pubmed). Ten studies that did not include either a healthy control group or a pre- and posttherapy contrast were excluded because without a basis for comparison any findings reported in such studies could not be attributed to SAD or treatment effect, respectively. For similar reasons, a study that used a mixed sample, and a study in which the SAD sample had neurodegenerative comorbidity, were also excluded. Finally, 2 studies that used region-of-interest-based techniques were excluded because of significant methodologic differences that make their comparability to other studies unreliable. These differences related to the variability (between subjects) of region-of-interest placement with only limited anatomic information (based on SPECT images only), as well as the fact that neither study spatially normalized participant scans to a standard template to ensure equivalence across subjects.

Nuclear neuroimaging experiments were broadly classified as those that investigated surrogates of neural activity (blood flow, perfusion, metabolism) and those that focused on imaging of functional neurochemistry.

\section{RESULTS}

Surrogates of Neural Activity (Blood Flow, Perfusion, Metabolism)

Meaningful appraisal of studies investigating surrogates of neural activity in SAD is challenging for several reasons. First, because the imaging tracers used in these experiments target different surrogates of neuronal activity (glucose metabolism or regional cerebral blood flow $[\mathrm{rCBF}]$ ), the correlations between modalities are not perfect. Second, differences in imaging paradigms (e.g., resting-state vs. task-based provocation) mean that the small number of existing studies must be evaluated in even smaller subgroups. Third, the analysis regions (whole brain vs. regional) and statistical thresholds used (voxel level vs. cluster level; corrections for multiple comparisons) vary widely. Finally, experiments into therapy effect are inconsistent in their choice of therapeutic intervention and mostly did not include placebo groups. Such study heterogeneity is not unique to nuclear neuroimaging experiments and is also frequently problematic in interpreting functional MRI (fMRI)-based work. Despite these drawbacks, nuclear imaging of neural activity has made some important contributions in SAD research.

Resting State. Current knowledge of resting regional neuronal activity in SAD is based largely on nuclear imaging experiments. Especially when interpreted together with resting-state-fMRI 
blood-oxygen-level-dependent data (which measure interregional correlations in resting state activity), such work is important because it establishes baseline conditions against which task-based research should be interpreted. No imaging studies investigating regional activity at rest have reported differences that are statistically significant at stringent statistical thresholds (e.g., corrected for multiple comparisons; $P<0.05$ ), suggesting that if such differences are present, they are probably small. At more liberal statistical thresholds, these studies provide evidence of disruptions in biologically plausible regions, including increases in several frontal regions, cerebellum (8), fusiform gyrus, and temporal pole (9) and decreases in subcallosal cortex and dorsal anterior cingulate cortex (10), as well as in pons, cerebellum, and precuneus (8). Investigating resting-state conditions using nuclear imaging with interregional correlations across subjects may be a more sensitive method to characterize abnormalities in SAD. A single nuclear imaging study that performed such analyses reported several disruptions (in SAD participants compared with healthy controls) significant at a stringent statistical threshold that were broadly consistent with results from fMRI work, namely increased connectivity between thalamus and middle frontal gyrus and decreased amygdala-frontal and posterior cingulate/precuneus-cerebellar connectivity (11).

Symptom Provocation. Increased amygdalar (and periamygdalar) reactivity in SAD during symptom provocation is a consistent finding in fMRI-based studies (7). It may therefore be surprising that results from similar experiments using ${ }^{15} \mathrm{O}$-water PET are variable in this respect (12-14). Possible reasons for this variability include the relatively small group sizes, differing thresholds for statistical significance, and the inferior spatial resolution of ${ }^{15} \mathrm{O}$ water PET. The largest of these studies, and the only one using a stringent statistical threshold, did not detect any difference in amygdalar reactivity between SAD and healthy control groups but found a correlation between amygdalar reactivity and the allelic status of serotonergic polymorphisms (14). There is no evidence from nuclear imaging studies of amygdalar hyperactivity during rest.

Therapy Effects. Limited evidence from nuclear imaging experiments in SAD suggests that various therapeutic interventions result in reductions in resting activity in limbic and paralimbic structures: a single dose of cannabidiol has been shown to reduce resting $\mathrm{rCBF}$ in parahippocampal gyrus and hippocampus (15), whereas in a combined group of SAD participants treated with either moclobemide or citalopram, bilateral reductions in $\mathrm{rCBF}$ were seen in insular cortex (16). A role for cingulate cortex in modulating treatment effects is supported by some of these experiments: cannabidiol has been shown to result in increased $\mathrm{rCBF}$ in the posterior cingulate

\section{NOTEWORTHY}

- The only research investigating resting regional neural activity (as opposed to connectivity) in SAD has been performed using nuclear imaging techniques.

- Evidence from functional neurochemistry imaging highlights dysfunction in the serotonergic and (possibly) the dopaminergic neurotransmitter systems in SAD.

- Future nuclear imaging research in SAD might focus on these and additional molecular targets of interest, such as exist in, for example, the oxytocinergic system, and make use of hybrid functional imaging such as PET/MRI.
(15) whereas functional connectivity work has demonstrated increases in rCBF-based connectivity of dorsal anterior cingulate cortex with precuneus and middle occipital gyrus (11). Finally, there is evidence that therapy results in altered glucose metabolism in the medial prefrontal cortex. In one experiment, after a course of tiagabine (a $\gamma$-aminobutyric acid reuptake inhibitor), SAD participants exhibited increased regional metabolism in the ventromedial prefrontal cortex (10), whereas in another experiment, moclobemide therapy was associated with reductions in metabolism in medial dorsal prefrontal cortex (9).

The effects of therapy on brain activity during task performance have also been studied in SAD. Compared with placebo, there is evidence that selective serotonin reuptake inhibitors, cognitive behavioral therapy, and the experimental neurokinin-1 antagonist vofopitant (GR205171) all result in reductions in rCBF to rhinal cortex, amygdala, hippocampal regions, and parahippocampal regions and that these reductions are greatest in responders $(17,18)$. Another experiment investigating the effect of nefazodone (a phenylpiperazine serotonin reuptake inhibitor and 5-hydroxytryptamine type 2 receptor antagonist) reported changes in $\mathrm{rCBF}$ during provocation in multiple regions, including reductions in precentral gyrus, insula, hypothalamus, and middle frontal and dorsal anterior cingulate cortices (13).

More detailed results of experiments that have investigated surrogates of neural activity in SAD compared with healthy controls, and the effect of therapy in SAD, are summarized in Supplemental Tables 1 and 2 and in Figure 1 (supplemental materials are available at http://jnm.snmjournals.org).

\section{Research into Functional Neurochemistry}

Serotonin. Genetic modification of serotonergic targets in mice has implicated the serotonergic system in anxiety and depression phenotypes (19). The importance of the serotonergic system in SAD is further underscored by the relative efficacy of selective serotonin reuptake inhibitors in treating the disorder (20). In vivo, nuclear imaging of the serotonin system is able to target both presynaptic and postsynaptic neurons. In presynaptic neurons, the rate of influx of ${ }^{11} \mathrm{C}$-5-hydroxytryptophan, a serotonin precursor, is a measure of the rate of serotonin synthesis. Studies using this tracer suggest that in SAD there are higher rates of serotonin synthesis in the hippocampus, basal ganglia, amygdala, and anterior cingulate $(21,22)$ and that synthesis decreases in the amygdala after therapy, with corresponding reductions in amygdalar $\mathrm{rCBF}$ on ${ }^{15} \mathrm{O}$-water PET (23). Serotonin synthesis rates in the amygdala and anterior cingulate have been linked with a specific genetic polymorphism (tryptophan-2 G-703T) in SAD (21). Another means of probing presynaptic serotonergic function is by imaging the serotonin transporter. The first such study, performed with ${ }^{11} \mathrm{C}-(6 R, 10 \mathrm{~b} S)-6$-(4-methylsulfanylphenyl)-1,2,3,5,6,10b-hexahydropyrrolo[2,1-a]isoquinoline $\left({ }^{11} \mathrm{C}-\mathrm{McN} 5652\right) \mathrm{PET}$, demonstrated increased occupancy of serotonin transporter after several months of paroxetine therapy (24). A study using another PET ligand, ${ }^{11} \mathrm{C}$-3-amino-4-(2-dimethylaminomethylphenylsulfanyl)-benzonitrile $\left({ }^{11} \mathrm{C}-\mathrm{DASB}\right)$, found that SAD participants have a higher serotonin transporter binding potential (reflecting higher serotonin transporter availability) than healthy controls in the raphe nuclei region, caudate nucleus, putamen, thalamus, and insular cortex (22). Only a single study has been performed investigating postsynaptic serotonergic function in SAD, in which researchers used ${ }^{11} \mathrm{C}-\mathrm{N}$-[2-[4-(2-methoxyphenyl)piperazin1 -yl]ethyl]- $N$-pyridin-2-ylcyclohexanecarboxamide $\left({ }^{11} \mathrm{C}\right.$-WAY100635) PET to measure binding of the serotonin $1 \mathrm{~A}$ receptor in 


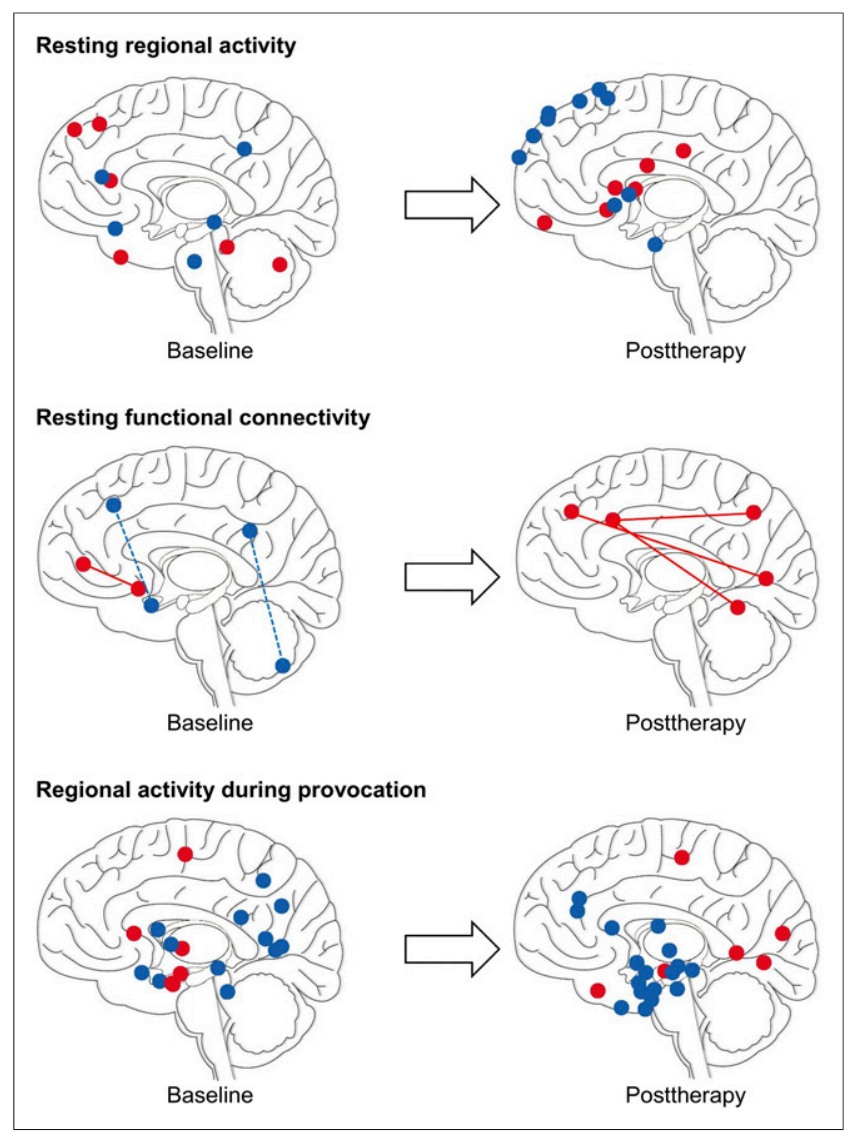

FIGURE 1. Changes in neural activity on nuclear imaging of SAD at baseline (left) and after therapy (right). Illustrations are for summary purposes only: sphere coordinates ( $y$ and $z$ ) roughly correspond to reported cluster maxima (Supplemental Table 1) and may appear deep or superficial to their true $x$ location. Red $=$ increased activity; blue $=$ decreased activity; solid line = increased resting functional connectivity; dashed line $=$ decreased resting functional connectivity. (Adapted from (60).)

SAD and healthy control participants (25). That group reported significantly lower binding potentials in several limbic and paralimbic regions, including amygdala, anterior cingulate, insula, and dorsal raphe nucleus in patients (Fig. 2). Binding potential in the hippocampus was not affected. In a later analysis of the same data, the researchers reported a negative correlation between serum cortisol levels and serotonin 1A receptor binding in amygdala, hippocampus, and retrosplenial cortex across all subjects, with stronger correlations in the amygdala and hippocampus in the SAD group (26).

Dopamine. The dopaminergic system has an established role in reward processing. Social interaction activates the reward system in ways similar to nonsocial rewards such as money, food, or addictive substances (27). The rewards achieved through personal interactions are thought to drive social affiliation. Consequently, the observation that SAD sufferers typically shun social interaction has led some researchers to propose that the biology of SAD may have a dopaminergic component. This proposal is also supported by several clinical observations (28), and several imaging studies have to date focused on the dopaminergic system in SAD. Similar to serotonergic targets, dopaminergic imaging can investigate both presynaptic neurons (using radiolabeled dopamine precursors, or tracers that target the dopamine transporter [DAT]) and postsynaptic neurons (with ligands that bind to $\mathrm{D}_{1}$ and $\mathrm{D}_{2} / \mathrm{D}_{3}$ dopamine receptors). All existing studies of presynaptic dopaminergic function in SAD used SPECT tracers that target DAT, such as ${ }^{123} \mathrm{I}$-ioflupane, ${ }^{123} \mathrm{I}-\beta$-CIT, and ${ }^{99 \mathrm{~m}} \mathrm{Tc}-2-[2-[[(1 S, 3 S, 4 R, 5 R)-3-$ (4-chlorophenyl)-8-methyl-8-azabicyclo[3.2.1]octan-4-yl]methyl(2-sulfanylethyl)amino]ethylamino]ethanethiol ( ${ }^{99 \mathrm{~m} T \mathrm{Tc}-T R O D A T-1) .}$ These studies are contradictory for striatal DAT binding in SAD participants compared with healthy controls, with work suggesting decreases (29), no difference (30), or increases (31) in the disorder. Postsynaptic dopaminergic research in SAD has focused on $\mathrm{D}_{2} / \mathrm{D}_{3}$ receptors and has been similarly inconclusive. The earliest study used ${ }^{123}$ I-iodobenzamide to image $\mathrm{D}_{2} / \mathrm{D}_{3}$ receptor binding and reported reduced striatal $\mathrm{D}_{2} / \mathrm{D}_{3}$ binding potential in SAD patients compared with controls but found no significant correlation with disease severity (32). Later studies by the same group (30) found no difference in striatal $\mathrm{D}_{2} / \mathrm{D}_{3}$ binding between $\mathrm{SAD}$ and healthy control groups using ${ }^{11} \mathrm{C}$-raclopride PET (an arguably more reliable technique). In a PET study using another $\mathrm{D}_{2} / \mathrm{D}_{3}$ receptor ligand, ${ }^{11} \mathrm{C}-(S)-(-)-5$-bromo- $N$ ((1-ethyl-2-pyrrolidinyl)methyl)-2,3-dimethoxybenzamide ( ${ }^{11} \mathrm{C}-\mathrm{FLB}$ 457), researchers confined their analysis to extrastriatal regions and reported increased $\mathrm{D}_{2} / \mathrm{D}_{3}$ binding in SAD in orbitofrontal cortex and dorsolateral prefrontal cortex (Fig. 3) (33). A study that used ${ }^{123}$ I-iodobenzamide SPECT to investigate dopaminergic function in SAD patients with comorbid obsessive-compulsive disorder reported dopaminergic deficits in patients with comorbidity, whereas those with obsessive-compulsive disorder alone demonstrated no differences from the healthy control group (34).

Effects of therapy on nuclear dopaminergic measures have also been investigated. In work investigating the effect of escitalopram on DAT binding, researchers reported a therapy-related increase in striatal DAT binding measured with ${ }^{123}$ I-ioflupane SPECT, although no correlation was found between symptom improvement and binding (35). Posttherapy increases in caudate DAT binding in that study were also seen in a subgroup of patients homozygous for

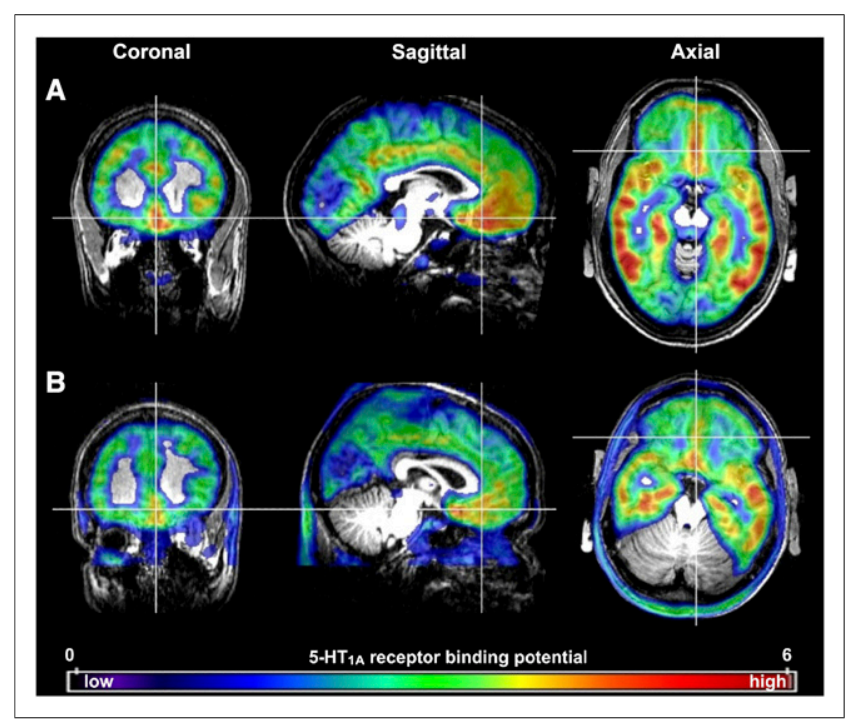

FIGURE 2. Serotonergic imaging using ${ }^{11} \mathrm{C}-\mathrm{WAY}-100635$. Parametric maps of serotonin receptor $1 \mathrm{~A}$ binding potential are superimposed on coregistered T1-weighted MR images of healthy subject $(A)$ and patient with SAD (B). White cross in triplanar view indicates corresponding area in mesial orbitofrontal cortex. $5-\mathrm{HT}_{1 \mathrm{~A}}=$ serotonin receptor $1 \mathrm{~A} ;{ }^{11} \mathrm{C}-\mathrm{WAY}-$ $100635={ }^{11} \mathrm{C}-\mathrm{N}-[2-[4-(2-$ methoxyphenyl)piperazin-1-yl] $]$ ethyl]- $N$-pyridin-2ylcyclohexanecarboxamide. (Adapted with permission of (25).) 




FIGURE 3. Dopaminergic imaging using ${ }^{11} \mathrm{C}-\mathrm{FLB} 457$. (Top) A priori constructed mask overlaid on Montreal Neurologic Institute template, which was used as search volume in analyses. (Middle and Bottom) Mean normalized parametric binding potential $\left(\mathrm{BP}_{\mathrm{ND}}\right)$ images of dopamine receptor type 2 availability for 16 healthy controls (HCs) and 12 patients (Pats) with SAD before mask was applied. Images are displayed in neurologic convention (right is right). (Adapted with permission of (33).)

the A10 DAT genotype. Another study investigated the effect of cognitive behavioral therapy on the interaction between disease severity and extrastriatal $\mathrm{D}_{2} / \mathrm{D}_{3}$ binding in $\mathrm{SAD}$ by performing ${ }^{11}$ C-FLB457 PET (36). After therapy, investigators reported a negative correlation between symptom reduction and $D_{2} / D_{3}$ binding in dorsolateral prefrontal cortex, medial prefrontal cortex, and hippocampus. This study was also noteworthy in that it reinforced the understanding that psychotherapy is an intervention that has effects on biology in many respects comparable to pharmacotherapy (37).

Conflicting results from these few nuclear imaging studies emphasize the need for further research to establish the role (if any) of dopamine in the disorder. Continued interest in dopamine is supported by several recent fMRI studies of the reward system in $\operatorname{SAD}(27,38)$.

Substance $P$. High concentrations of the neurokinin-1 receptor are expressed in several limbic structures (39). It has been suggested that substance $\mathrm{P}$, which binds to this receptor, may play an important role in anxiety disorders (40). A single study that used the PET radiotracer ${ }^{11} \mathrm{C}-(2 S, 3 S)-N$-[ [2- ${ }^{11} \mathrm{C}$-methoxy-5-[5-(trifluoromethyl)tetrazol-1-yl]phenyl]methyl]-2-phenyl-piperidin-3-amine $\left({ }^{11} \mathrm{C}-\mathrm{GR} 205171\right)$ to image neurokinin-1 receptor binding in SAD demonstrated that compared with healthy controls, binding in the amygdala was higher in the disorder (41).

A summary of molecular imaging targets studied in SAD is presented in Supplemental Table 3. An illustration summarizing the findings of nuclear imaging experiments that targeted neurotransmitter systems in SAD is found in Figure 4.

\section{DISCUSSION}

Much is already known about the neuroanatomy relevant in social anxiety (42). Neuroimaging research using both fMRI and nuclear techniques further supports functional models of SAD that show increased neuronal activity in limbic and paralimbic regions during anxiety provocation, with reductions in anxiety leading to attenuation of this hyperreactivity. Further, both fMRI and nuclear methods have provided data on disruptions in resting brain networks in the disorder. PET and SPECT have made important contributions to knowledge of resting regional brain activity and have found abnormalities in several key limbic and paralimbic regions, as well as in medial frontal cortex.

Knowledge of regional neuronal activity under baseline conditions is important for two reasons. First, it enables improved interpretation of disruptions in resting-state functional connectivity, typically studied using resting-state fMRI. For example, a reduction in resting functional connectivity between two regions may be due to unmatched increases or decreases in neuronal activity in either or both regions. Although it is relatively simple to identify interregional correlations at rest, measuring the regional changes in neuronal activity that drive these correlations is more difficult with commonly used fMRI methods. Second, knowledge of both resting regional activity and resting functional connectivity

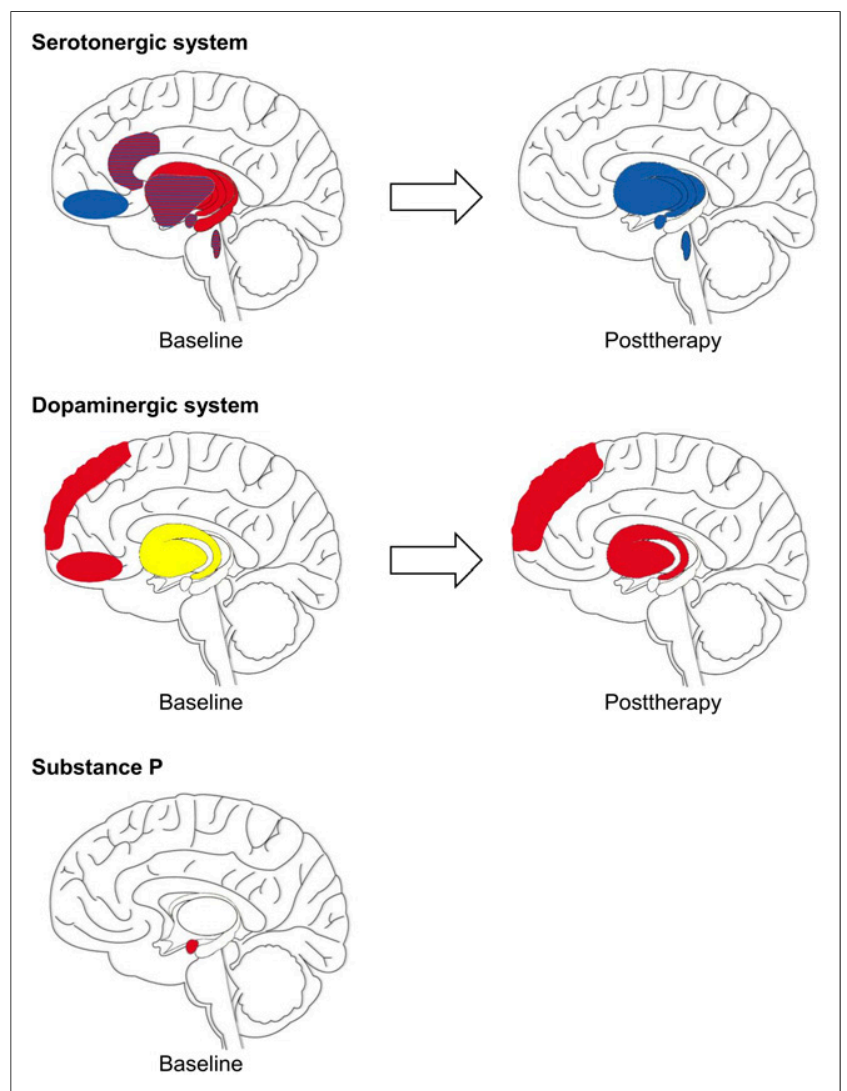

FIGURE 4. Changes in neurotransmitter systems on nuclear imaging of SAD at baseline (left) and after therapy (right). Illustration is for summary purposes only: shaded regions $(y$ and $z$ ) may appear deep or superficial to their true $x$ location. Red $=$ increased activity; blue $=$ decreased activity; red-blue stripes $=$ both increased and decreased activity, depending on molecular target; yellow = conflicting evidence. (Adapted from (60).) 
is important for contextualizing the results of task-based experiments. It has, for example, been shown that by integrating subjectspecific structural and resting-state functional neuroimaging data, it is possible to predict regional brain activations in response to a variety of tasks (43). Importantly, this shifts the focus from observed differences in how participants process information to disturbances in functional network architecture that underlie these differences (43). Nuclear techniques are useful in delineating the complex relationships between resting regional activity, resting connectivity, and task-based reactivity.

Within regions involved in SAD, understanding of the underlying neurochemistry will be key, and it is in this regard that nuclear imaging techniques will likely make their greatest contribution. The integration of data, obtained from nuclear imaging experiments investigating functional neurochemistry, into biologic models of SAD is particularly challenging for several reasons. First, the scarcity of such data reduces their reliability and the likelihood of representativeness. Second, it is frequently impossible to determine causality when biologic disruptions are identified in anxious samples. Third, anxiety disorders as a group share many common features, making it demanding to identify features unique to SAD. Finally, the endophenotype of SAD, which must incorporate genetic and environmental influences with the interactions of multiple neurotransmitter systems that give rise to variant neural activations and ultimately to symptoms and behavior, is likely to be extremely complex. More than one such endophenotype may explain the heterogeneity of the disorder (44).

Despite these challenges, some progress has been made. There are four major neurotransmitters identified as potentially important in anxiety, that is, $\gamma$-aminobutyric acid, noradrenaline, serotonin and, dopamine (28), of which only the latter two have been investigated in SAD.

Signaling mechanisms via serotonin receptors (of which at least 14 have been identified) are diverse and have the potential to modulate both excitatory ( $\gamma$-aminobutyric acid-ergic) and inhibitory (glutamatergic) neurotransmitter systems (45). Of all serotonergic receptors, subtype $1 \mathrm{~A}$ is probably the best studied and of particular interest to depression and anxiety research given its high concentrations in limbic and paralimbic structures (including amygdala, anterior cingulate, posterior cingulate, subcallosal area, insula, and temporal pole) (46). In this light, findings of variable reductions in binding of serotonergic receptor subtype $1 \mathrm{~A}$ in several of these regions in SAD are intriguing (25). Accepting the role of serotonin as modulatory, with both excitatory and inhibitory downstream functions, raises the possibility that variations in the concentration, distribution, or function of a variety of serotonin receptors may underlie the spectrum of changes in neural activity within these regions, and in regions with which they are functionally connected, as represented in network models of SAD (7). This possibility has been previously explored $(19,23,47)$ and is supported by other work that has linked variations in the serotonin transporter gene to variations in amygdalar reactivity and its functional connectivity in non-SAD samples $(48,49)$.

The dopaminergic system includes both excitatory $\left(D_{1}\right.$ and $\left.D_{5}\right)$ and inhibitory $\left(D_{2}-D_{4}\right)$ receptors and has also received attention based on several clinical observations that have linked dopamine to SAD. These include the observations that social anxiety is a common symptom of Parkinson disease and chronic stimulant abuse, both of which are associated with presynaptic dopaminergic degeneration; reports of dopamine agonists causing SAD; and reports of successful treatment of SAD with drugs that increase dopamine availability (28). It is also noteworthy that one of the principal dopaminergic pathways, the mesolimbic pathway, is involved with reward processing, including the processing of social reward (50). The observation that people with SAD tend to avoid social interactions (and the rewards they may confer) has led some investigators to hypothesize that disrupted reward processing may be a feature of the condition (51). There is emerging evidence from fMRI work that this is indeed the case $(27,38)$, although exactly which components of reward processing are involved is still unclear. In this context, the as yet limited and inconsistent results from nuclear imaging studies that have investigated dopaminergic targets (DAT and the inhibitory $\mathrm{D}_{2} / \mathrm{D}_{3}$ family of receptors) in SAD are difficult to reconcile. Research focusing on alternative dopaminergic targets such as the $D_{1}$ family of receptors (which are excitatory) may be interesting. It is also possible that (with the exception of SAD caused by dopaminergic degeneration), rather than being characterized by a dopaminergic deficit per se, SAD is associated with disruptions in neurotransmitter systems that modulate dopaminergic function. Such neurotransmitters include the serotonergic system (52), for which there is stronger evidence of abnormality in SAD, as well as the endocannabinoid and oxytocinergic systems, both of which have been implicated in the experience of social reward $(53,54)$.

It is also noteworthy that nuclear imaging of neurotransmitter targets in SAD has to date focused entirely on the resting state. Research using PET imaging to study functional neurochemistry during task performance is also possible (55) and might be worthwhile to conduct in SAD. Such research may prove key in our understanding of the behavioral correlates of functional neuroimaging measures. Beyond the systems presented in this review, there are multiple other molecular tracers (e.g., targeting $\gamma$-aminobutyric acid and noradrenaline receptors) that present interesting research subjects in SAD. In addition, progress is being made on the development of a PET tracer that is selective for the oxytocin receptor in the brain (56). This would raise the prospect of in vivo imaging of central oxytocin action in SAD, which is of considerable interest given the putative role of this peptide hormone in social anxiety and social cognition (57). Ultimately, research using new ligands has the potential to identify novel molecular targets for future drug development.

Nuclear studies of rCBF and metabolism have provided new insights into how anxiolytics change regional neuronal activity both at rest and during symptom provocation in SAD. More than this, imaging techniques targeting components of various neurotransmitter systems have identified changes in functional neurochemistry after therapy. Such findings are relevant to our understanding of the neurobiology of SAD and the mechanisms by which drugs exert their anxiolytic properties and are relevant for drug discovery. Although some early work suggests that certain nuclear imaging features may predict individual patients' responses to a specific treatment, there is currently insufficient evidence that these features can be applied clinically. The possibility of personalized medicine based on molecular imaging results is a tantalizing prospect, and it is hoped that more research will lead to progress in this regard.

Two further key developments may soon have an impact on the role of nuclear imaging methods in SAD research. The first is the recent advent of hybrid PET/MRI systems. The ability to image MRI signal simultaneously with dynamic imaging of a wide range of molecular targets, both at rest and during tasks, may transform functional neuroimaging research. The benefit of synchronous 
measurement is that it allows for the direct temporal correlation between processes imaged by fMRI (such as neural activity) and those imaged by PET (such as neurotransmitter kinetics). This direct correlation is impossible with sequential multimodal imaging because scan conditions differ. A second important development is the establishment of shared data repositories, providing open access to researchers. Initiatives such as the Human Connectome Project and the United Kingdom Biobank (which currently include only MRI-based neuroimaging data) have enabled mega analyses with vastly increased statistical power. Large databanks also allow researchers to use normative modeling, a technique that maps cohort heterogeneity along a continuum rather than the (frequently artificial) clustering used in traditional case-control studies, which assume homogeneity within cohort groupings (58). Similar benefits are seen from the trend toward large-scale, international collaborations such as the Evidence-Based Network for the Interpretation of Germline Mutant Alleles (ENIGMA) consortium (59). The future inclusion of nuclear imaging data in these or similar repositories and collaborations would potentially add great value to new research initiatives, especially when studied in combination with phenotyping and genotyping data.

\section{CONCLUSION}

Nuclear neuroimaging techniques represent powerful tools, complementary to MRI-based techniques, to investigate brain function and the neurobiologic effects of therapy. This is true both of the study of SAD and of neuroscience research in general. Although basic models of the fear response are now well characterized, research into both regional activity and interregional relationships of diverse brain regions outside the limbic and paralimbic circuits must still be conducted. Preliminary nuclear imaging evidence of disrupted neurochemistry in SAD requires further study to draw more definitive conclusions and to approach a foundation for designing treatments based on targeting individuals' molecular signatures. The development of novel brain tracers, the advent of hybrid PET/MRI systems, and the growth of open-access data repositories that include nuclear imaging contributions all lend promise to further improvements in SAD research.

\section{DISCLOSURE}

Dan Stein and Christine Lochner receive funding from the South African Medical Research Council. Alexander Doruyter was funded by the South African Medical Research Council under the MRC Clinician Researcher Programme. Publication costs were funded by the Nuclear Technologies in Medicine and the Biosciences Initiative (NTeMBI), managed by the South African Nuclear Energy Corporation (Necsa) and funded by the Department of Science and Technology of South Africa. No other potential conflict of interest relevant to this article was reported.

\section{REFERENCES}

1. Anxiety disorders. In: Diagnostic and Statistical Manual of Mental Disorders: DSM-5. Arlington, VA: American Psychiatric Association; 2013:202-203.

2. Stein DJ, Lim CCW, Roest AM, et al. The cross-national epidemiology of social anxiety disorder: data from the World Mental Health Survey Initiative. BMC Med. 2017;15:143.

3. Wittchen HU, Beloch E. The impact of social phobia on quality of life. Int Clin Psychopharmacol. 1996;11(suppl 3):15-23.
4. Fox AS, Kalin NH. A translational neuroscience approach to understanding the development of social anxiety disorder and its pathophysiology. Am J Psychiatry. 2014;171:1162-1173.

5. Etkin A, Wager TD. Functional neuroimaging of anxiety: a meta-analysis of emotional processing in PTSD, social anxiety disorder, and specific phobia. Am J Psychiatry. 2007;164:1476-1488.

6. Freitas-Ferrari MC, Hallak EC, Trzesniak C, et al. Neuroimaging in social anxiety disorder: a systematic review of the literature. Prog Neuropsychopharmacol Biol Psychiatry. 2010;34:565-580.

7. Brühl AB, Delsignore A, Komossa K, Weidt S. Neuroimaging in social anxiety disorder: a meta-analytic review resulting in a new neurofunctional model. Neurosci Biobehav Rev. 2014;47:260-280.

8. Warwick JM, Carey P, Jordaan GP, Dupont P, Stein DJ. Resting brain perfusion in social anxiety disorder: a voxel-wise whole brain comparison with healthy control subjects. Prog Neuropsychopharmacol Biol Psychiatry. 2008;32:1251-1256.

9. Doruyter A, Dupont P, Taljaard L, Stein DJ, Lochner C, Warwick JM. Resting regional brain metabolism in social anxiety disorder and the effect of moclobemide therapy. Metab Brain Dis. 2018;33:569-581.

10. Evans KC, Simon NM, Dougherty DD, et al. A PET study of tiagabine treatment implicates ventral medial prefrontal cortex in generalized social anxiety disorder. Neuropsychopharmacology. 2009;34:390-398.

11. Doruyter A, Lochner C, Jordaan GP, Stein DJ, Dupont P, Warwick JM. Resting functional connectivity in social anxiety disorder and the effect of pharmacotherapy. Psychiatry Res Neuroimaging. 2016;251:34-44.

12. Tillfors M, Furmark T, Marteinsdottir I, et al. Cerebral blood flow in subjects with social phobia during stressful speaking tasks: a PET study. Am J Psychiatry. 2001;158:1220-1226.

13. Kilts CD, Kelsey JE, Knight B, et al. The neural correlates of social anxiety disorder and response to pharmacotherapy. Neuropsychopharmacology. 2006;31:2243-2253.

14. Furmark T, Henningsson S, Appel L, et al. Genotype over-diagnosis in amygdala responsiveness: affective processing in social anxiety disorder. $J$ Psychiatry Neurosci. 2009;34:30-40.

15. Crippa JAS, Derenusson GN, Ferrari TB, et al. Neural basis of anxiolytic effects of cannabidiol (CBD) in generalized social anxiety disorder: a preliminary report. J Psychopharmacol. 2011;25:121-130.

16. Warwick JM, Carey P, Van der Linden G, et al. A comparison of the effects of citalopram and moclobemide on resting brain perfusion in social anxiety disorder. Metab Brain Dis. 2006;21:241-252.

17. Furmark T, Appel L, Michelgård A, et al. Cerebral blood flow changes after treatment of social phobia with the neurokinin-1 antagonist GR205171, citalopram, or placebo. Biol Psychiatry. 2005;58:132-142.

18. Furmark T, Tillfors M, Marteinsdottir I, et al. Common changes in cerebral blood flow in patients with social phobia treated with citalopram or cognitive-behavioral therapy. Arch Gen Psychiatry. 2002;59:425-433.

19. Albert PR, Vahid-Ansari F, Luckhart C. Serotonin-prefrontal cortical circuitry in anxiety and depression phenotypes: pivotal role of pre- and post-synaptic 5HT1A receptor expression. Front Behav Neurosci. 2014;8:199.

20. Ipser JC, Kariuki CM, Stein DJ. Pharmacotherapy for social anxiety disorder: a systematic review. Expert Rev Neurother. 2008;8:235-257.

21. Furmark T, Marteinsdottir I, Frick A, et al. Serotonin synthesis rate and the tryptophan hydroxylase-2: G-703T polymorphism in social anxiety disorder. J Psychopharmacol. 2016;30:1028-1035.

22. Frick A, Åhs F, Engman J, et al. Serotonin synthesis and reuptake in social anxiety disorder: a positron emission tomography study. JAMA Psychiatry. 2015;72: 794-802.

23. Frick A, Åhs F, Appel L, et al. Reduced serotonin synthesis and regional cerebral blood flow after anxiolytic treatment of social anxiety disorder. Eur Neuropsychopharmacol. 2016;26:1775-1783.

24. Kent JM, Coplan JD, Lombardo I, et al. Occupancy of brain serotonin transporters during treatment with paroxetine in patients with social phobia: a positron emission tomography study with ${ }^{11} \mathrm{C} \mathrm{McN}$ 5652. Psychopharmacology (Berlin). 2002;164:341-348.

25. Lanzenberger RR, Mitterhauser M, Spindelegger C, et al. Reduced serotonin-1A receptor binding in social anxiety disorder. Biol Psychiatry. 2007;61:1081-1089.

26. Lanzenberger R, Wadsak W, Spindelegger C, et al. Cortisol plasma levels in social anxiety disorder patients correlate with serotonin-1A receptor binding in limbic brain regions. Int J Neuropsychopharmacol. 2010;13:1129-1143.

27. A Richey J, Ghane M, Valdespino A, et al. Spatiotemporal dissociation of brain activity underlying threat and reward in social anxiety disorder. Soc Cogn Affect Neurosci. 2017;12:81-94.

28. Durant C, Christmas D, Nutt D. The pharmacology of anxiety. In: Behavioral Neurobiology of Anxiety and Its Treatment. Berlin, Germany: Springer; 2010: 303-330. 
29. Tiihonen J, Kuikka J, Bergström K, Lepola U, Koponen H, Leinonen E. Dopamine reuptake site densities in patients with social phobia. Am J Psychiatry. 1997;154:239-242.

30. Schneier FR, Abi-Dargham A, Martinez D, et al. Dopamine transporters, D2 receptors, and dopamine release in generalized social anxiety disorder. Depress Anxiety. 2009;26:411-418.

31. van der Wee NJ, van Veen JF, Stevens H, van Vliet IM, van Rijk PP, Westenberg HG. Increased serotonin and dopamine transporter binding in psychotropic medication-naive patients with generalized social anxiety disorder shown by ${ }^{123}$ I-beta-(4-iodophenyl)-tropane SPECT. J Nucl Med. 2008;49:757-763.

32. Schneier FR, Liebowitz MR, Abi-Dargham A, Zea-Ponce Y, Lin SH, Laruelle M. Low dopamine D2 receptor binding potential in social phobia. Am J Psychiatry. 2000;157:457-459.

33. Plavén-Sigray P, Hedman E, Victorsson P, et al. Extrastriatal dopamine D2-receptor availability in social anxiety disorder. Eur Neuropsychopharmacol. 2017;27:462-469.

34. Schneier FR, Martinez D, Abi-Dargham A, et al. Striatal dopamine $\mathrm{D}_{2}$ receptor availability in OCD with and without comorbid social anxiety disorder: preliminary findings. Depress Anxiety. 2008;25:1-7.

35. Warwick JM, Carey PD, Cassimjee N, et al. Dopamine transporter binding in social anxiety disorder: the effect of treatment with escitalopram. Metab Brain Dis. 2012;27:151-158.

36. Cervenka S, Hedman E, Ikoma Y, et al. Changes in dopamine D2-receptor binding are associated to symptom reduction after psychotherapy in social anxiety disorder. Transl Psychiatry. 2012;2:e120.

37. Linden DEJ. How psychotherapy changes the brain: the contribution of functional neuroimaging. Mol Psychiatry. 2006;11:528-538.

38. Cremers HR, Veer IM, Spinhoven P, Rombouts SARB, Roelofs K. Neural sensitivity to social reward and punishment anticipation in social anxiety disorder. Front Behav Neurosci. 2015;8:439.

39. Ribeiro-da-Silva A, Hökfelt T. Neuroanatomical localisation of substance $P$ in the CNS and sensory neurons. Neuropeptides. 2000;34:256-271.

40. Ebner K, Muigg P, Singewald G, Singewald N. Substance P in stress and anxiety: NK-1 receptor antagonism interacts with key brain areas of the stress circuitry. Ann N Y Acad Sci. 2008;1144:61-73.

41. Frick A, Ahs F, Linnman C, et al. Increased neurokinin-1 receptor availability in the amygdala in social anxiety disorder: a positron emission tomography study with [ ${ }^{11}$ C]GR205171. Transl Psychiatry. 2015;5:e597.

42. Miskovic V, Schmidt LA. Social fearfulness in the human brain. Neurosci Biobehav Rev. 2012;36:459-478.

43. Tavor I, Jones OP, Mars RB, Smith SM, Behrens TE, Jbabdi S. Task-free MRI predicts individual differences in brain activity during task performance. Science. 2016;352:216-220.
44. Bas-Hoogendam JM, Blackford JU, Brühl AB, Blair KS, van der Wee NJA, Westenberg PM. Neurobiological candidate endophenotypes of social anxiety disorder. Neurosci Biobehav Rev. 2016;71:362-378.

45. Olivier B. Serotonin: a never-ending story. Eur J Pharmacol. 2015;753:2-18.

46. Varnäs K, Halldin C, Hall H. Autoradiographic distribution of serotonin transporters and receptor subtypes in human brain. Hum Brain Mapp. 2004;22:246260.

47. Garcia-Garcia AL, Newman-Tancredi A, Leonardo ED. P5-HT1A receptors in mood and anxiety: recent insights into autoreceptor versus heteroreceptor function. Psychopharmacology (Berlin). 2014;231:623-636.

48. Munafò MR, Brown SM, Hariri AR. Serotonin transporter (5-HTTLPR) genotype and amygdala activation: a meta-analysis. Biol Psychiatry. 2008;63:852857.

49. Pezawas L, Meyer-Lindenberg A, Drabant EM, et al. 5-HTTLPR polymorphism impacts human cingulate-amygdala interactions: a genetic susceptibility mechanism for depression. Nat Neurosci. 2005;8:828-834.

50. Rilling J, Gutman D, Zeh T, Pagnoni G, Berns G, Kilts C. A neural basis for social cooperation. Neuron. 2002;35:395-405.

51. Mathew SJ, Coplan JD, Gorman JM. Neurobiological mechanisms of social anxiety disorder. Am J Psychiatry. 2001;158:1558-1567.

52. Esposito E, Di Matteo V, Di Giovanni G. Serotonin-dopamine interaction: an overview. Prog Brain Res. 2008;172:3-6.

53. Harari-Dahan O, Bernstein A. A general approach-avoidance hypothesis of oxytocin: accounting for social and nonsocial effects of oxytocin. Neurosci Biobehav Rev. 2014;47:506-519.

54. Wei D, Allsop S, Tye K, Piomelli D. Endocannabinoid signaling in the control of social behavior. Trends Neurosci. 2017;40:385-396.

55. Ceccarini J, Vrieze E, Koole M, et al. Optimized in vivo detection of dopamine release using ${ }^{18}$ F-fallypride PET. J Nucl Med. 2012;53:1565-1572.

56. Wenzel B, Mollitor J, Deuther-Conrad W, et al. Development of a novel nonpeptidic ${ }^{18} \mathrm{~F}$-labeled radiotracer for in vivo imaging of oxytocin receptors with positron emission tomography. J Med Chem. 2016;59:1800-1817.

57. Kirsch P. Oxytocin in the socioemotional brain: implications for psychiatric disorders. Dialogues Clin Neurosci. 2015;17:463-476.

58. Marquand AF, Rezek I, Buitelaar J, Beckmann CF. Understanding heterogeneity in clinical cohorts using normative models: beyond case-control studies. Biol Psychiatry. 2016;80:552-561.

59. Thompson PM, Andreassen OA, Arias-Vasquez A, et al. ENIGMA and the individual: predicting factors that affect the brain in 35 countries worldwide. Neuroimage. 2017;145:389-408.

60. Brains, bodies, and behavior. In: Introduction to Psychology. Minneapolis, MN: University of Minnesota Libraries Publishing; 2010. 